OPEN ACCESS

Edited by:

Djordje Miljkovic,

University of Belgrade, Serbia

Reviewed by:

Stefano Ugel,

University of Verona, Italy

Alain Le Moine,

Free University of Brussels, Belgium

*Correspondence:

Jordi Ochando

jochando@isciii.es

Specialty section: This article was submitted to Immunological Tolerance and Regulation,

a section of the journal

Frontiers in Immunology

Received: 14 December 2018 Accepted: 14 February 2019 Published: 06 March 2019

Citation:

Ochando J, Conde P, Utrero-Rico A and Paz-Artal E (2019) Tolerogenic Role of Myeloid Suppressor Cells in

Organ Transplantation.

Front. Immunol. 10:374. doi: 10.3389/fimmu.2019.00374

\section{Tolerogenic Role of Myeloid Suppressor Cells in Organ Transplantation}

\author{
Jordi Ochando ${ }^{1,2 \star}$, Patricia Conde ${ }^{1,2}$, Alberto Utrero-Rico ${ }^{3}$ and Estela Paz-Artal ${ }^{3,4}$ \\ ${ }^{1}$ Department of Medicine, Icahn School of Medicine at Mount Sinai, New York, NY, United States, ${ }^{2}$ Immunología de \\ Trasplantes, Centro Nacional de Microbiología, Instituto de Salud Carlos III, Madrid, Spain, ${ }^{3}$ Grupo de Inmunodeficiencias e \\ Inmunología del Trasplante, Instituto de Investigación Sanitaria Hospital 12 de Octubre (imas12), Madrid, Spain, ${ }^{4}$ School of \\ Medicine, Complutense University, Madrid, Spain
}

Myeloid-derived suppressor cells (MDSC) are a heterogeneous population of immature cells of myeloid origin with a specific immune inhibitory function that negatively regulates the adaptive immune response. Since MDSC participate in the promotion of tolerance in the context of organ transplantation, therapeutic strategies that regulate the induction and development of MDSC have been the center of scientist attention. Here we review literature regarding induction of MDSC with demonstrated suppressive function among different types of allografts and their mechanism of action. While manipulation of MDSC represents a potential therapeutic approach for the promotion of donor specific tolerance in solid organ transplantation, further characterization of their specific phenotype, which distinguishes MDSC from non-suppressive myeloid cells, and detailed evaluation of the inhibitory mechanism that determines their suppressive function, is necessary for the realistic application of MDSC as biomarkers in health and disease and their potential use as immune cell therapy in organ transplantation.

\section{Keywords: MDSC, immune tolerance, transplantation, immunophenotyping, myeloid cells}

\section{INTRODUCTION}

Myelopoiesis is a regular process where the cells of the mononuclear phagocyte system (MPS) originate from common myeloid precursors (CMP) leading to monocytes, macrophages and dendritic cells (DC) under steady state. Under acute pathological conditions, myeloid cells respond to immunogenic signals like PAMPs (pathogen-associated molecular patterns) or DAMPs (damage-associated molecular patterns) showing multiple protective immune functions such as phagocytosis, pro-inflammatory cytokine secretion and activation of $\mathrm{T}$ cells. Under chronic pathological conditions, such as persistent inflammation or certain malignancies, myeloid cells are stimulated by continue immunogenic signals that have important effects on cell differentiation (1). The standard pathway for CMP cells differentiation is inhibited and the myelopoiesis is altered under chronic inflammation, which results in undifferentiated myeloid cells (2). Myeloid derived suppressor cells (MDSC) represent a mixture of myeloid progenitor cells at different stages of differentiation that may develop into macrophages, DC or granulocytes depending the microenvironment $(3,4)$.

Since the discovery of MDSC there has been an effort to define their heterogeneity, origin and function beyond cancer relates studies. The scientific interest of these cells have been spread through many fields where the immune system is altered due to chronic pathological conditions, 
such as graft vs. host disease (GvHD), organ transplantation, infection, and autoimmune diseases. To better define the heterogeneity, murine MDSC were initially defined as myeloid cells expressing CD11b and GR-1. Based on these two markers and their morphology, MDSC were divided into two major groups: Granulocytic MDSC (G-MDSC) and monocytic MDSC (M-MDSC). Several studies included the differential expression of inducible nitric oxide synthase (iNOS) and arginase (Arg) and subdivided MDSC population based on the expression of Ly6C and Ly6G (5). CD11b+Ly6ChiLy6G- MDSC have a monocytic-like morphology express nitric oxide synthase 2 (NOS2), have increased $\mathrm{T}$ cell suppressive activity and are identified as M-MDSC. In contrast, CD11b+Ly6ClowLy6G+ MDSC have a granulocyte-like morphology and express high levels of arginase type 1 (Arg1) (5). Some authors recommend the term polymorphonuclear (PMN)-MDSC instead of G-MDSC attending to the differences in the phenotype from steady-state neutrophils. PMN-MDSC show less granules, reduced CD16 and CD62L and increased Arg1, peroxynitrite and CD11b expression (6). Additional MDSC populations have been described based on the intensity of the Gr-1 gene expression as Gr-1lo, Gr-1int, and Gr-1hi (7). Based on recent findings showing differences in modulation of the cell death pathway, the anti-apoptotic markers c-FLIP and MCL-1 could be also of help to respectively distinguish M-MDSC and granulocytic MDSC subsets. Different from granulocytic MDSC, the continuous expression of c-FLIP is needed by M-MDSC survival and function and defines them as the dominant immunosuppressive subset. This observation points out modulation of c-FLIP in monocytes to promote or block immunosuppressive cells for therapy purposes $(8,9)$.

The overlapping expression of phenotypic markers makes also difficult to distinguish MDSC from tumor-associated macrophages (TAM) and tumor-associated neutrophils (TAN). Mouse M-MDSC are phenotypically described as CD11b+Ly6ChiLy6G- myeloid cells expressing low levels of F4/80, while TAM express high levels of F4/80 (4). Human MDSC were initially described in cancer patients as lineage negative CD34+ cells (10). Thereafter, other myeloid markers such as the human leucocyte antigen (HLA)-DR was identified in a renal cell carcinoma study to define human MDSC as $\mathrm{CD} 33+\mathrm{CD} 11 \mathrm{~b}+\mathrm{HLA}-\mathrm{DR}-$ (11). Additionally, the use of CD14 expression is accepted for human M-MDSC, although it is still controversial for PMN-MDSC since granulocytes express low levels of this marker. As a result, M-MDSC are defined as CD33+CD11b+HLA-DR-CD14+ while PMNMDSC are defined as CD33+CD11b+CD15+CD66b+ (6). Recently, another MDSC subpopulation was described as CD33+CD11b+HLA-DR-CD14-CD15-CD66b-, including a mixture of immature cells named early stage MDSC (e-MDSC). Recent findings suggest that PMN- and M-MDSC are the most potent immunosuppressive cells while e-MDSC show less Arg1 and iNOS amounts and may not inhibit $\mathrm{T}$ cells proliferation. More studies are needed to understand if e-MDSC are true MDSC precursors and evaluate their clinical significance (12). It is also recommended the use of additional phenotypic markers, such as CD62L, CD16 and the vascular endothelial growth-factor receptor1 (VEGFR1) to better define human MDSC (13).
Phenotypic characterization of MDSC remains controversial as MDSC are described as myeloid cells in different stages of differentiation associated with immune-regulatory molecules and receptors (Arg1, NOS2/NO, NOX2/ROS, PD-L1, and VEGF2), transcription factors (S100a8 and STAT3) and cytokines (IL10 , TGF $\beta$ and IL4-R) (6). Since phenotypic characterization of MDSC is still debatable, MDSC are better defined as potent immunosuppressive myeloid cells characterized by less phagocytic activity or the production of high levels of reactive oxygen and nitrogen species and anti-inflammatory cytokines (14). The capacity to modulate $\mathrm{T}$ cells activity is the most often used immune suppressive feature of MDSC, which is also associated with their increased capacity to induce $\mathrm{T}$ cell apoptosis (15) and expansion of regulatory T cells (Treg) (16). Although immune modulation of $\mathrm{T}$ conventional cell activity is probably the main reported function of MDSC, the interaction between MDSC and other immune cells has been described in recent years. These include suppression of the $\mathrm{B}$ cells (17), dysregulation of $\mathrm{T}$ follicular helper cells $(18,19)$, loss of natural killer cell (NK) function (11) and suppression of DC development (20).

Focusing on the mechanism of MDSC induction, inhibitors of the mammalian target of rapamycin (mTOR), which represents a major immunosuppressive drugs for organ transplant recipients (21), plays a crucial role in promoting the development of MDSC. Using an immunological hepatic injury model, it was demonstrated rapamycin served as a functional immune modulator of $\mathrm{CD}_{11 b^{+}} \mathrm{Grl}^{+}$MDSC (22). Mechanistically, the authors demonstrated that mTOR down-regulation promotes $\mathrm{CD}_{11 \mathrm{~b}}{ }^{+} \mathrm{Grl}^{+} \mathrm{Ly}_{6} \mathrm{Chi}^{\mathrm{iNOS}}{ }^{+} \mathrm{M}-\mathrm{MDSC}$ recruitment to the inflammatory site that produced NO for tissue repair. Rapamycin also enhanced the suppressive function in murine PMN-MDSC after bone marrow transplantation, via up-regulation of $\operatorname{Arg} 1$ and iNOS (23). However, the effects of rapamycin and mTOR inhibition on MDSC remains controversial, as transgenic mice with a myeloid-specific deletion of mTOR display a decreased the number of M-MDSC in vivo after skin allograft transplantation (24).

\section{MDSC IN ORGAN TRANSPLANTATION}

The mononuclear phagocyte system (MPS), comprising DC, monocytes and macrophages, is implicated in many immunological mechanisms occurring during recognition of the non-self and the alloimmune response against the transplanted organ (25). Recipient DC infiltrate the allograft and form cognate contacts with $\mathrm{T}$ cells promoting effector $\mathrm{T}$ cell mediated rejection (26). In addition, donor DC derived exosomes promote an alloimmune response against the allograft by transferring functional MHC molecules to recipient DC (27). Acquisition of exosomes activates recipient DC that present donor MHC molecules to alloreactive $\mathrm{T}$ cells promoting $\mathrm{T}$ cell immunity. Monocytes also play a critical role in organ transplantation as they mediate the immune response against allogeneic nonself (28) and initiate allograft rejection by inducing $\mathrm{T}$ cell mediated immune responses (29). Macrophages act as effectors 
of tissue damage in acute renal allograft rejection (30) and represent the majority of cells that infiltrate an allograft under severe rejecting conditions (31). Mechanistically, activated graft infiltrating macrophages increase their aerobic glycolysis metabolism and secrete pro-inflammatory cytokines associated with acute rejection (32). In addition to the MPS, neutrophils also play a critical role during organ rejection. The Lakkis laboratory demonstrated that depletion of neutrophils with anti-Ly6G significantly decreased inflammatory alloresponses (28). This is consistent with previous observations, which suggested that early neutrophil influx into the transplanted allograft favors organ rejection (33). Mechanistically, neutrophils may contribute to allograft rejection by different pathways that include the secretion of inflammatory cytokines (34), B cell stimulation (35) and through antigen presentation to T cells (36).

Since DC, monocytes, macrophages and neutrophils all the myeloid contribute to organ transplant rejection, MDSC must therefore prevent their immunogenicity against the allograft. Consequently, therapeutic protocols that prolong organ transplant survival may induce the development of MDSC, which inhibit myeloid cell derived graft reactive immune responses, such as antigen presentation and lymphocyte activation. Alternatively, experimental approaches that promote organ transplant acceptance may skew the differentiation of immunogenic DC, monocyte, macrophage and neutrophil precursors toward M-MDSC and G-MDSC favoring immune tolerance (Figure 1). Below we describe the role of MDSC in different organ transplant settings.

\section{Kidney Transplantation}

Vanhove's laboratory was the first to report the role of MDSC in kidney transplant recipient rats (37). In this experimental model, tolerance was induced by a costimulatory blockade with anti CD28 antibody. Myeloid cells expressing $\mathrm{CD} 11 \mathrm{~b}^{+} \mathrm{CD} 80 / 86^{+} \mathrm{Sirp} \alpha^{+}$accumulated in the recipient allograft and were defined as MDSC for the first time in the context of organ transplantation. This study indicated that $\mathrm{CD} 11 \mathrm{~b}^{+} \operatorname{Sirp} \alpha^{+}$ MDSC isolated from blood and bone marrow were able to suppress proliferation of anti CD3 anti-CD28 stimulated T cells. This suppressive mechanism of tolerance was in part mediated by iNOS, which was upregulated in graft infiltrating MDSC and by blood MDSC upon co-culture with activated effector $\mathrm{T}$ cells but not in Treg. The mechanistic role of NO in MDSCmediated suppression was initially described by Mazzoni and colleagues using a NO synthase knockout mice (38). The authors demonstrated that $\mathrm{CD}_{11 b^{+} \mathrm{Gr}-1^{+}}$MDSC from the spleens of immunosuppressed mice inhibit $\mathrm{T}$ cell proliferation in a NOdependent manner, in response to signals from activated $\mathrm{T}$ cells that included IFN- $\gamma$. Another report from Vanhove's laboratory indicated that secretion of CCL5 by graft infiltrating MDSC was responsible for the accumulation of Treg into tolerized kidney allografts (39). In subsequent studies, Dilek and colleagues analyzed blood MDSC gene expression from kidney recipient showing that CCL5 was strongly downregulated after tolerant regimen. The amount of intra graft CCL5 protein was unchanged (40). The results indicate that a gradient of CCL5 between the graft and peripheral blood might contribute to the intra graft localization of Treg in tolerant recipients controlled by MDSC.

In the clinical setting, Hock and coworkers showed significantly increased frequencies of total MDSC (CD33 ${ }^{\text {pos }}$ HLA-DR ${ }^{\text {neg }}$ cells into the blood low density fraction), as well as both $\mathrm{CD} 14^{\text {pos }}$ and CD14 ${ }^{\text {neg }}$-MDSC subsets in renal transplant recipients relative to normal donors. MDSC subsets frequencies and MDSC/DC ratios were higher in kidney recipients with or without current or prior squamous cell carcinoma than in healthy controls. In vitro, fMLP-activated MDSC from transplanted patients inhibited T cell proliferation (41). MDSC were shown to expand early after transplantation, independently of using basiliximab or thymoglobulin during induction (42) (and our unpublished observations).

Consistent with data from Vanhove's laboratory describing the presence of MDSC and of Treg in kidney transplanted rats, data from Murphy and colleagues reported presence of MDSC in human kidney transplant recipients. The study evaluated the capacity of blood derived $\mathrm{CD} 11 \mathrm{~b}^{+} \mathrm{CD} 33^{+} \mathrm{HLA}-$ $\mathrm{DR}^{-}$MDSC to suppress $\mathrm{CD} 4^{+} \mathrm{T}$ cells proliferation in vitro. In addition, blood derived MDSC were able to expand Treg in vitro and correlated with increased Treg numbers in vivo (43). This was the first study where MDSC were associated with Treg in human transplant recipients. Consistent with these results, ex vivo experiments performed by Hoechst and colleagues demonstrated that blood derived CD $14^{+} \mathrm{HLA}^{-\mathrm{DR}}{ }^{--}$ myeloid cells isolated from hepatocellular carcinoma (HCC) patients induce $\mathrm{CD} 4{ }^{+} \mathrm{CD} 25^{+}$Foxp $3^{+}$Treg when co-cultured with autologous $\mathrm{T}$ cells (44). A recent report from this group demonstrated that, while $\mathrm{CD} 14^{+} \mathrm{HLA}^{-D R^{-}}$MDSC induce Foxp $^{+}$Treg, CD $14^{+}$HLA-DR $^{+}$myeloid cells from the same patient promote the generation of pathogenic Th17 cells when co-cultured with naive CD $4^{+} \mathrm{T}$ cells. Importantly CD $14^{+} \mathrm{HLA}-$ $\mathrm{DR}^{-}$MDSC modulate the trans differentiation of Foxp $3^{+}$Treg from monocyte-induced Th17 cells in a TGF- $\beta$ and retinoic acid (RA) dependent mechanism (45).

More recently, the expression of myeloid-related S100A8 and S100A9 proteins was analyzed in two independent cohorts of patients with acute rejection. These proteins showed in vitro suppressive properties including inhibition of DC maturation and enhancement of ROS production. High S100A8 and S100A9 mRNA levels in biopsies predicted improved graft outcome and both proteins expression correlated with MDSC markers into PBMC and renal biopsies. Intragraft, high amounts of S100A9 correlated with lower expression of $\mathrm{T}$ cell immunity (CD3 $\varepsilon$ and CD4) and increased FoxP3, IL-10, and TGF- $\beta$ regulatory markers (46). In a retrospective cohort of 50 renal recipients with biopsy-proven acute $\mathrm{T}$-cell mediated rejection, patients with high

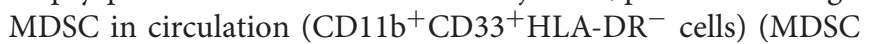
$>10 \%$ into $\mathrm{PBMC}$ ) showed increased estimated glomerular filtration rate and lower serum creatinine at the time of biopsy. Compared to low MDSC recipients, high MDSC patients showed less development of IFTA and significantly superior 1- and 5-year graft survival (47). However, prospective and randomized studies in large cohorts are still lacking to better understand the role of MDSC in clinical kidney transplant and their potential value as biomarker or therapy target. 


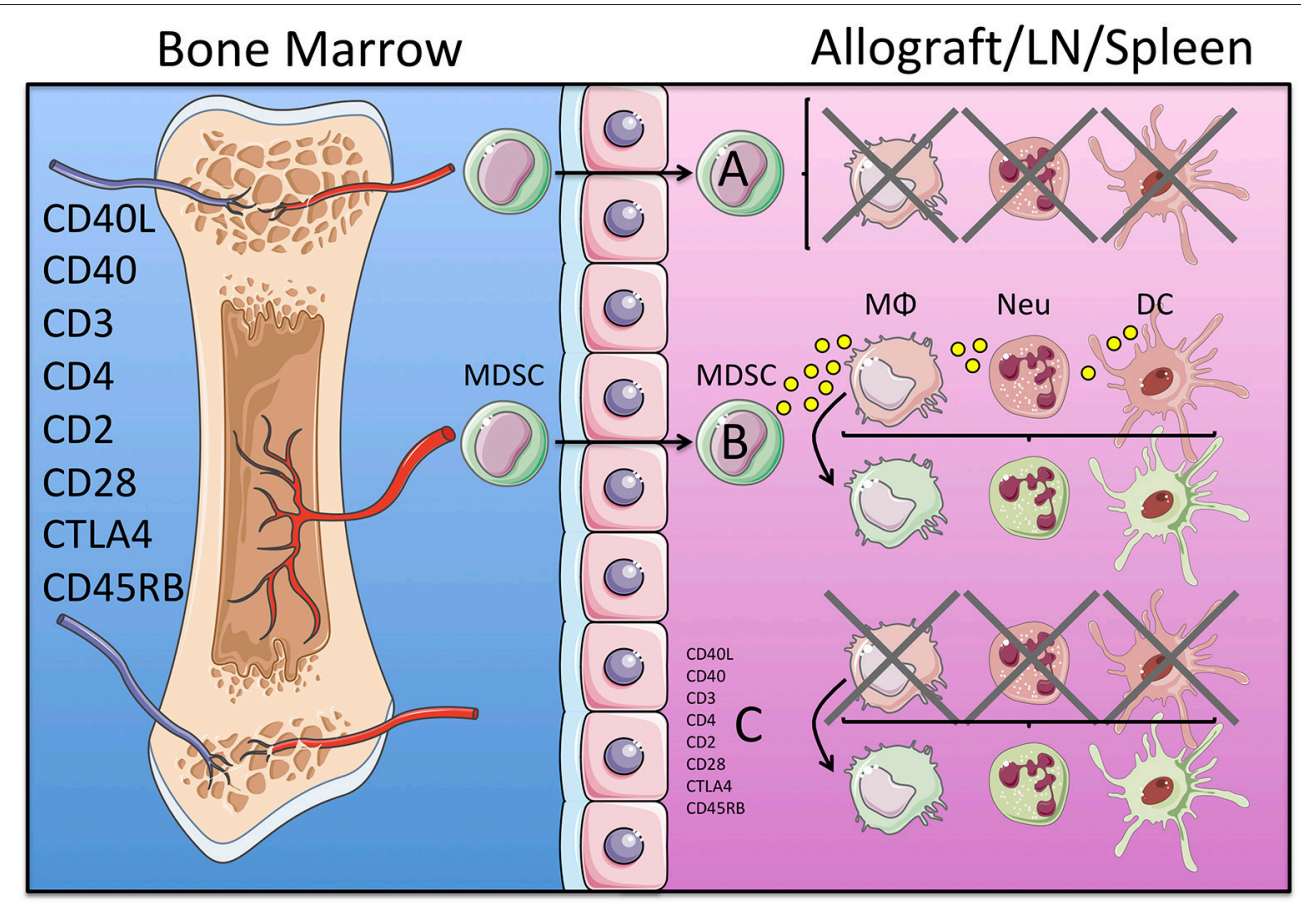

FIGURE 1 | Potential mechanisms of immune regulation mediated by MDSC in organ transplantation. Induction of transplantation tolerance in experimental murine models is achieved by targeting TCR and co-stimulatory blockade with monoclonal antibodies. These therapeutic treatments may induce the development of an MDSC precursor that leaves the bone marrow and may migrate into the allograft, lymph node (LN) and/or the spleen. Once in the tissue MDSC may mediate direct inhibition of immunogenic myeloid cells (macrophages, neutrophils and dendritic cells in red), as depicted in (A); or secrete cytokines and growth factors that convert immunogenic (red) into tolerogenic (green) myeloid cells, as depicted in (B). Alternatively, both processes (direct inhibition of immunogenic and/or conversion into tolerogenic myeloid cells) may be a direct effect of the tolerogenic regimen (monoclonal antibodies) independently of the MSDC, as depicted in (C).

\section{Corneal Transplantation}

Corneal allograft models in mice have been used to test the use or manipulation of MDSC as immunomodulatory strategies in transplantation. In two groups of mice receiving either cornea or cornea and skin allografts, longer grafts survival was observed in animals with prior transference of bone marrow MDSC induced in cecal ligated and punctured mice (48). In a different experimental model, B6 mice corneas were transplanted into $\mathrm{BALB} / \mathrm{c}$ recipients who received intraperitoneal dexamethasone (dex) at decreasing doses from day 0 to 21 after surgery. Administration of dex significantly prolonged the allograft survival and correlated with decreased infiltration of $\mathrm{CD} 3+$ cells and low levels of IFN $-\gamma$ and IL- $1 \beta$ in the grafts, together with low IFN- $\gamma$ CD4+ cells in draining lymph nodes, blood, spleen and bone marrow. Concomitantly, an expansion of MHC class $\mathrm{II}^{-} \mathrm{CD} 11 \mathrm{~b}^{+} \mathrm{Ly}_{6 \mathrm{C}} \mathrm{C}^{+}$monocytes (m-MDSC following Bronte and coworkers, ref 6) and increased iNOS were observed in the same compartments of dex-treated mice. FACS-sorted $\mathrm{CD}_{11 \mathrm{~b}^{+}} \mathrm{Ly} 6 \mathrm{C}^{+}$cells from bone marrow of dex-treated mice inhibited in vitro $\mathrm{CD} 4+\mathrm{T}$ cells proliferation and prolonged the survival of corneal allografts when transferred into dexuntreated recipients. Depletion of $\mathrm{MHC}$ class $\mathrm{II}^{-} \mathrm{CD} 11 \mathrm{~b}^{+} \mathrm{Ly}_{6 \mathrm{C}^{+}}$ monocytes abrogated the protective effect of dex on corneal allografts suggesting that these cells were required for mediating the induction of tolerance by glucocorticoids (49). Similar results were obtained in corneal allograft recipient mice treated with a rapamycin nano-micelle (RNM) ophthalmic solution. Under this therapy, delay of rejection and expansion of $\mathrm{Gr} 1^{\text {int }} \mathrm{CD} 11 \mathrm{~b}^{+}$ MDSCs in allografts, cervical lymph nodes, blood and spleen were observed. The capacity of MDSCs from the RNM solutiontreated mice to suppress proliferation of $\mathrm{CD} 4+\mathrm{T}$ cells depended on iNOS and arginase-1, and the administration of anti-Gr-1 antibody or the pharmacological inhibition of iNOS abrogated the beneficial effects of rapamycin (50).

\section{Pancreatic Islets Transplantation}

In the Bronte's pioneer work murine MDSCs were obtained by treating bone marrow in vitro with GM-CSF + IL-6. When adoptively transferred into islet-allografted syngeneic mice, the GM-CSF + IL-6 induced MDSCs increased the survival of functional islets. Integrity of the $\mathrm{C} / \mathrm{EBP} \beta$ transcription factors was needed to develop the tolerogenic MDSC (51).

In different experimental settings, prolonged islets allograft survival has been achieved by MDSC generated by hepatic stellate cells (HSC). CCR2 expression was needed to allow migration of HSC-induced MDSC into allografts (52), and their suppressive capacity relied on induction of apoptosis in $\mathrm{T}$ effector cells and B7-H1-mediated expansion of Tregs $(53,54)$. The induction of MDSC by HSC depended from soluble factors. Interestingly, SDS-PAGE and LC-MS analysis of the most bioactive fraction in 
hepatic stellate cells culture supernatant identified complement component 3 (C3) as a key mediator, since depletion of HSCderived C3 markedly reduced the ability to induce MDSC (53). Moreover, co-transplantation of $\mathrm{BALB} / \mathrm{c}$ mice islets with wild type or $\mathrm{C}^{-/-}$HSC into diabetic B6 mice showed that $60 \%$ of mice receiving wild type HSC remained normoglycemic by post-transplant day 60 while all recipients of C3-deficient HSC lost islets under day 21. Draining lymph nodes and grafts from wild type HSC recipients showed increased frequency of Tregs and CD11c $\mathrm{c}^{-}$myeloid cells of immature phenotype, which were able to suppress proliferation of $\mathrm{CD}^{+}$and $\mathrm{CD} 8^{+}$cells and production of IFN $\gamma$ (55). However, results in the opposite direction were obtained in a model of streptozotocin-induced diabetes in mice, in which expansion of MDSC and protection of pancreas against the autoimmune destruction were noticed in the absence of C3, and depletion of MDSC by anti-Gr1 led to diabetes in $\mathrm{C}^{-/-}$streptozotocin-treated mice (56). Concluding results to fully understand if and how $\mathrm{C} 3$ is required to promote generation of MDSC are still missing.

\section{Skin Transplantation}

Using an experimental skin transplant model where bm12 MHC-II minor mismatched graft were transplanted into C57BL/6 recipients, the Horuzsko laboratory demonstrated HLD-G expressing MDSC interacts with immune inhibitory receptors, such as immunoglobulin-like transcript 2 (ILT2), which induces the expansion of MDSC in vivo (57). The authors further demonstrated that MDSC from ILT2 transgenic mice exhibit an augmented suppressive function and were able to prolong skin graft survival following adoptive transfer into C57BL/6 recipients.

De Wilde and colleagues demonstrated that repetitive administration of lipopolysaccharide (LPS) in vivo induces the development $\mathrm{CD}_{11 \mathrm{~b}}{ }^{+} \mathrm{Gr}-1^{+}$MDSC (58). In a male-to-female mismatched skin transplantation model, the authors reported that in vivo transfer of MDSC treated with LPS significantly prolonged skin allograft survival. This was due to the expression of heme oxygenase-1 (HO-1), which impaired $\mathrm{T}$ cell activation. The authors also demonstrated that LPS induced MDSC suppresses Th1 and Th2 cytokine production, while produce large amounts of IL-10 as suppressive mechanism. Further, HO1 inhibition by a specific inhibitor completely abolished T-cell suppression and IL-10 production. The importance of HO1 during prolonged allograft survival was first described by Yamashita et al. (59). The authors demonstrated that cobalt protoporphyrin IX (CoPPIX) treatment leads to a significant upregulation $\mathrm{HO}-1$ that was necessary for indefinite survival of fully mismatched heart allografts.

In a recent study, Zhao et al. established that the combination of M-CSF and TNF $\alpha$ efficiently induces functional MDSC in vitro (60). The resulting M-MDSC were characterized by the expression of F4/80, CD80, and PD-L1. Mechanistically, M$\mathrm{CSF}+\mathrm{TNF} \alpha$ induced M-MDSC upregulated the expression of iNOS, which was necessary for suppression of $\mathrm{T}$ cell proliferation. Upon adoptive transfer, M-CSF+TNF $\alpha$ induced M-MDSC promoted immune tolerance in male-to-female skin transplanted mice. Consistent with Vanhove's observation, blockade of iNOS activity failed to induce the graft acceptance, demonstrating that immunosuppressive ability of M-CSF+TNF $\alpha$-induced MMDSC is dependent on iNOS. The critical role of iNOS activity in the suppressive function of MDSC was also described by $\mathrm{Wu}$ et al. (61). The authors identified Smad3 as an intrinsic negative regulator of MDCS development and recognized that the immunosuppressive function of MDCS depends on NO production. Using Smad3 deficient mouse recipients in a fully mismatched skin transplantation model, the authors observed an increase in both granulocytic and monocytic cells associated with less production of anti-donor IgG Abs and decreased IFN- $\gamma$ production. Interestingly, L-NMMA significantly reduced NO production and efficiently blocked the immunosuppressive effects of Smad3-deficient G-MDSC on T cell proliferation.

In agreement with these results, Liao and colleagues demonstrated that MDSC development is induced by dexamethasone through the glucocorticoid receptor (GR) pathway $(62,63)$. Dexamethasone treatment significantly prolonged allograft survival in a fully allogeneic skin transplant model in mice through upregulation of iNOS and NO production in MDSC. These results validate the administration of glucocorticoids as a therapeutic approach that prolongs graft survival through the development of MDSC.

Further studies have also illustrated the immune-modulatory activity of IL-33 during the induction of iNOS expressing MDSC (64). Using both syngeneic and allogeneic skin transplants models, Pino-Lagos and colleagues demonstrated that IL33 treatment up-regulated the number of Foxp3 ${ }^{+}$Treg and promoted the conversion of Foxp $3^{-}$T cells into Foxp $3^{+}$Treg in the periphery.

\section{Heart Transplantation}

Using a mouse heart transplantation model Rodriguez-Garcia and colleagues demonstrated the requirement of MDSC for the induction of transplantation tolerance (65). The authors treated recipient mice with anti-CD40L mAb costimulatory blockade and identified the critical role for tolerogenic $\mathrm{CD} 11 \mathrm{~b}^{+} \mathrm{Ly}_{6 \mathrm{C}} \mathrm{Cow}^{\text {low }}$ expressing MDSC. Using depletional mAbs, clodronate-loaded liposomes, and transgenic mice specific for depletion of CD11 $\mathrm{b}^{+}$ expressing cells the authors reported that monocytic precursors migrate from the bone marrow to the transplanted organ early after transplantation and prevent the initiation of adaptive immune responses that lead to allograft rejection.

Similar results were previously obtained from Terry Strom laboratory (66), which demonstrated that $\mathrm{CD} 11 \mathrm{~b}^{+} \mathrm{Gr} 1^{\text {low }} \mathrm{MDSC}$ exhibit high suppressive capacities and prevent grafts from prolonged cold ischemia-mediated injury. The authors induced the development of Ly6C ${ }^{\text {low }}$ MDSC by treating fully allogeneic recipient mice with rapamycin $(3 \mathrm{mg} / \mathrm{kg})$ and costimulatory blockade with anti-CD40L mAb. This combination therapy induced tolerance in C57BL/6 mice pre-sensitized by Balb/c skin grafts at day-7 that received $\mathrm{BALB} / \mathrm{c}$ heart grafts in contrast to mice treated with either rapamycin or anti-CD40L mAb alone.

Consistent with these results, a recent report from Braza and colleagues described a novel nanoimmunotherapy based on highdensity lipoprotein (HDL) that targets myeloid cell precursors in vivo (32). Using a fully allogeneic mouse heart transplant 
model, the authors demonstrated that a rapamycin and CD40 costimulatory blockade combined nanotherapy (mTORi/Traf6iHDL) favored the accumulation of $\mathrm{CD} 11 \mathrm{~b}^{+} \mathrm{Ly} 6 \mathrm{C}^{\text {low }}$ myeloid cells, which prevented $\mathrm{CD} 8^{+} \mathrm{T}$ cell proliferation and promoted Treg expansion. Remarkably, a short-term treatment with nanobiologics during the first week after transplantation resulted in indefinite allograft survival of most transplant recipients with no signs of chronic rejection.

Considering mTOR inhibitors as a current immunosuppressive therapy for organ transplantation, Nakamura and colleagues demonstrated that treatment with rapamycin results in a significant prolongation of graft survival mediated by iNOS expressing MDSC in a murine cardiac transplantation model (67). The authors also confirmed that $\mathrm{CD}_{11 \mathrm{~b}^{+}}$myeloid cells expressing lower levels of Gr-1 efficiently suppressed $\mathrm{CD}^{+} \mathrm{T}$ cell proliferation in vitro. Interestingly, adoptive transfer of rapamycin-induced MDSC and to a lesser extent G-MDSC, through the coronary arteries before organ reperfusion of transplant recipient mice significantly prolonged allograft survival. Graft survival prolongation of MDSC was associated with an increase of splenic Foxp $3^{+}$Treg. Mechanistically, the Nakamura group further reported that rapamycin treatment induces the expression of PD-L1 on MDSC that accumulate in the cardiac allograft following adoptive transfer (68).

A critical aspect in organ transplantation is the induction of donor-specific unresponsiveness. For this purpose, many authors use donor specific splenocytes in combination with tolerogenic therapy, such as co-stimulatory blockade $(69,70)$. In this respect, Luo and colleagues performed infusions of donor splenocytes treated with 1-ethyl-3-(3'-dimethylaminopropyl)-carbodiimide (ECDI-SPs) before and after transplantation $(71,72)$. The authors observed prolonged allograft survival associated with intragraft accumulation of $\mathrm{CD}_{11 \mathrm{~b}}{ }^{+}$MDSC that express high levels of indoleamine 2,3 dioxygenase (IDO). Furthermore, combination therapy of donor ECDI-SPs with systemic rapamycin induced indefinite cardiac allograft survival in $100 \%$ of the recipients for over 150 days.

\section{Liver, Bowel, and Lung Transplantation}

Whereas, liver transplants can be spontaneously accepted without the requirement of immunosuppression in different species $(73,74)$, the immune response acutely rejects hepatocyte transplants (75). This suggests that liver stromal cells protect parenchymal cells from rejection. Hepatic stellate cells have potent immune regulatory activity and they have been shown to promote MDSCs generation in vivo and in vitro $(53,76)$.

Kim et al. showed that in Rhesus macaques MDSC accumulate in high numbers in the liver when compared to blood, spleen and lymph nodes (77). In a model of allogeneic orthotopic liver transplantation in rats, the authors observed that the promotion of tolerance by treatment with rapamycin was associated with an increase of regulatory $\mathrm{T}$ cell phenotypes and accumulation of MDSC in spleen (78).

In a prospective cohort of 36 intestinal transplant recipients, the authors identified MDSC (lineage ${ }^{-} \mathrm{HLADR}^{-/ \text {low }} \mathrm{CD}_{3} 3^{+} \mathrm{CD} 11 \mathrm{~b}^{+}$-expressing cells) and all three M- $\left(\mathrm{CD} 14^{+} \mathrm{CD} 15^{-}\right)$, PMN- $\left(\mathrm{CD} 14^{-} \mathrm{CD} 15^{+}\right)$and e-MDSC populations into PBMC. All three MDSCs subsets increased post-transplant although PMN-MDSC and e-MDSC did so immediately, while M-MDSC increased after 2 months posttransplant. All three MDSC types were able to suppress CD4+ and $\mathrm{CD} 8+\mathrm{T}$ cells proliferation as well as IFN $\gamma$ production. High plasma levels of IL- 6 but not TNF $\alpha$ or GM-CSF, the use of exogenous steroids and low tacrolimus trough levels correlated with MDSCs numbers in PBMC. In agreement, IL-6 and methylprednisolone enhanced MDSC cells after culturing bone marrow cells from healthy controls in basic medium with GM-CSF and G-CSF. Intragraft MDSCs were low before transplantation but increased during a year after transplantation. The analysis of chemokines expression in intestinal grafts biopsies and of chemokines receptors expression in MDSC supported a role for CCL11 and CCL15 in recruiting CCR1and CCR3- expressing M- MDSCs and e-MDSCs, and a role for CXCL6 in recruiting CXCR2- expressing PMN- MDSCs and eMDSCs into the mucosa of intestinal allografts. Peripheral blood MDSCs were significantly lower in patients with acute rejection. The addition of MDSC into co-cultures of donor-reactive T cells with donor-derived intestinal epithelial organoids enhanced the organoids viability, suggesting that the accumulation of MDSC suppressed $\mathrm{T}$ cells alloresponse against the donor intestinal epithelium (79).

In the only publication regarding lung transplant at present, the authors observed that the phyla Firmicutes dominated the microbiome signature in the distal airways of subjects without bronchiolitis obliterans syndrome (BOS), while this shifted to a Proteobacteria-dominant signature in the BOS cohort. Suppressive MDSC predominated in the proximal airways and pro-inflammatory myeloid cells were more abundant in distal airways. These results suggested a functional link between the local microbiome and MDSC phenotype, which may play a role in the pathogenesis of BOS (80).

\section{CONCLUSION AND PERSPECTIVES}

Regulation of different MDSC subsets with distinct immune function may be used for future therapeutic approach that promotes tolerance in organ transplantation. In the setting of murine GVHD, the inflammasome activation in the transferred MDSC induced the loss of their suppressive capacity, thus, understanding the micro environmental signals that affect the stability of the MDSC suppressive capacity will be also critical for an optimal use in therapy (81). In addition, MDSC may be used as biomarkers that provide critical information regarding the functional immune status of organ transplant recipients. Noninvasive immune approaches that determine and characterize MDSC subsets in humans are urgently needed to move the field forward. Unfortunately, identification of specific mechanisms by which MDSC exhibit suppressive functions and contribute to the development of tolerance remains a difficult task. One of the main difficulties resides in a consensual classification and identification MDSC subset during pathological conditions. For that purpose, the COST action Mye-EUNITER was established in 
2014 (http://www.mye-eunit er.eu/) to create a general consensus to standardize the function and phenotype of MDSC across different species (82). Additional immune regulatory molecules, such as B7-H3 (83), may be validated in future experiments to further identify and characterize MDSC in transplant recipients. In this respect, the use of next generation genomic sequencing may help to identify the transcriptomic profile of different MDSC subsets and differentiate suppressor cells from normal myeloid cells (84).

One critical consideration to fully determine whether MDSC are indeed functionally inhibitory myeloid cell is the choice of the immune functional assay (85). While the clinical application of MDSC represents a promising therapeutic approach for the induction of organ transplant acceptance either as a cell therapy or by regulating there in vivo development from myeloid precursors, it requires consensus on markers that identify MDSC subsets, which limit our ability to specifically target MDSC in vivo.

\section{REFERENCES}

1. Libby P. Inflammatory mechanisms: the molecular basis of inflammation and disease. Nutr Rev. (2007) 65:S140-6. doi: 10.1301/nr.2007.dec.S140-S146

2. Gabrilovich DI, Bronte V, Chen SH, Colombo MP, Ochoa A, OstrandRosenberg S, et al. The terminology issue for myeloid-derived suppressor cells. Cancer Res. (2007) 67:425. doi: 10.1158/0008-5472.CAN-06-3037

3. Movahedi K, Laoui D, Gysemans C, Baeten M, Stange G, Van den Bossche J, et al. Different tumor microenvironments contain functionally distinct subsets of macrophages derived from Ly6C(high) monocytes. Cancer Res. (2010) 70:5728-39. doi: 10.1158/0008-5472.CAN-09-4672

4. Sawanobori Y, Ueha S, Kurachi M, Shimaoka T, Talmadge JE, Abe $\mathrm{J}$, et al. Chemokine-mediated rapid turnover of myeloid-derived suppressor cells in tumor-bearing mice. Blood. (2008) 111:5457-66. doi: 10.1182/blood-2008-01-136895

5. Youn JI, Nagaraj S, Collazo M, Gabrilovich DI. Subsets of myeloid-derived suppressor cells in tumor-bearing mice. J Immunol. (2008) 181:5791-802. doi: 10.4049/jimmunol.181.8.5791

6. Bronte V, Brandau S, Chen SH, Colombo MP, Frey AB, Greten $\mathrm{TF}$, et al. Recommendations for myeloid-derived suppressor cell nomenclature and characterization standards. Nat Commun. (2016) 7:12150. doi: $10.1038 /$ ncomms 12150

7. Dolcetti L, Peranzoni E, Ugel S, Marigo I, Fernandez Gomez A, Mesa C, et al. Hierarchy of immunosuppressive strength among myeloid-derived suppressor cell subsets is determined by GM-CSF. Eur J Immunol. (2010) 40:22-35. doi: 10.1002/eji.200939903

8. Fiore A, Ugel S, De Sanctis F, Sandri S, Fracasso G, Trovato R, et al. Induction of immunosuppressive functions and NF-kappaB by FLIP in monocytes. Nat Commun. (2018) 9:5193. doi: 10.1038/s41467-018-0 7654-4

9. Haverkamp JM, Smith AM, Weinlich R, Dillon CP, Qualls JE, Neale $\mathrm{G}$, et al. Myeloid-derived suppressor activity is mediated by monocytic lineages maintained by continuous inhibition of extrinsic and intrinsic death pathways. Immunity. (2014) 41:947-59. doi: 10.1016/j.immuni.2014. 10.020

10. Pak AS, Wright MA, Matthews JP, Collins SL, Petruzzelli GJ, Young MR. Mechanisms of immune suppression in patients with head and neck cancer: presence of CD34(+) cells which suppress immune functions within cancers that secrete granulocyte-macrophage colony-stimulating factor. Clin Cancer Res. (1995) 1:95-103.

11. Li H, Han Y, Guo Q, Zhang M, Cao X. Cancer-expanded myeloid-derived suppressor cells induce anergy of NK cells through membrane-bound TGFbeta 1. J Immunol. (2009) 182:240-9. doi: 10.4049/jimmunol.182.1.240

\section{AUTHOR CONTRIBUTIONS}

All authors listed have made a substantial, direct and intellectual contribution to the work, and approved it for publication.

\section{FUNDING}

This work was supported by COST (European Cooperation in Science and Technology) Actions: BM1305: Action to Focus and Accelerate Cell Tolerogenic Therapies (A FACTT), and BM1404: European Network of Investigators Triggering Exploratory Research on Myeloid Regulatory Cells (Mye-EUNITER), the Spanish Ministry of Science SAF201680031-R (JO), the Comunidad de Madrid B2017/BMD-3731 (JO), the project 13/00045 (Instituto de Salud Carlos III, Spain, cofounded by the European Regional Development Fund) and project IDEAS206PAZ (Asociación Española contra el Cáncer) to EP-A.

12. Lang S, Bruderek K, Kaspar C, Hoing B, Kanaan O, Dominas N, et al. Clinical relevance and suppressive capacity of human myeloidderived suppressor cell subsets. Clin Cancer Res. (2018) 24:4834-44. doi: 10.1158/1078-0432.CCR-17-3726

13. Nagaraj S, Gabrilovich DI. Myeloid-derived suppressor cells in human cancer. Cancer J. (2010) 16:348-53. doi: 10.1097/PPO.0b013e3181eb3358

14. Youn JI, Collazo M, Shalova IN, Biswas SK, Gabrilovich DI. Characterization of the nature of granulocytic myeloid-derived suppressor cells in tumorbearing mice. J Leukoc Biol. (2012) 91:167-81. doi: 10.1189/jlb.0311177

15. Bronte V, Wang M, Overwijk WW, Surman DR, Pericle F, Rosenberg SA, et al. Apoptotic death of CD8+ T lymphocytes after immunization: induction of a suppressive population of Mac-1+/Gr-1+ cells. J Immunol. (1998) 161:531320.

16. Chesney JA, Mitchell RA, Yaddanapudi K. Myeloid-derived suppressor cellsa new therapeutic target to overcome resistance to cancer immunotherapy. $J$ Leukoc Biol. (2017) 102:727-40. doi: 10.1189/jlb.5VMR1116-458RRR

17. Green KA, Cook WJ, Green WR. Myeloid-derived suppressor cells in murine retrovirus-induced AIDS inhibit T- and B-cell responses in vitro that are used to define the immunodeficiency. J Virol. (2013) 87:2058-71. doi: 10.1128/JVI.01547-12

18. Wang C, Zhang N, Qi L, Yuan J, Wang K, Ma S, et al. Myeloidderived suppressor cells inhibit $\mathrm{T}$ follicular helper cell immune response in japanese encephalitis virus infection. J Immunol. (2017) 199:3094-105. doi: 10.4049/jimmunol.1700671

19. Wang L, Cao D, Zhao J, Nguyen LN, Dang X, Ji Y, et al. HCV-associated exosomes promote myeloid-derived suppressor cell expansion via inhibiting miR-124 to regulate T follicular cell differentiation and function. Cell Disc. (2018) 4:51. doi: 10.1038/s41421-018-0052-z

20. Poschke I, Mao Y, Adamson L, Salazar-Onfray F, Masucci G, Kiessling R. Myeloid-derived suppressor cells impair the quality of dendritic cell vaccines. Cancer Immunol. Immunother. (2012) 61:827-38. doi: 10.1007/s00262-011-1143-y

21. Kajiwara M, Masuda S. Role of mTOR Inhibitors in kidney disease. Int J Mol Sci. (2016) 17:975. doi: 10.3390/ijms 17060975

22. Zhang Y, Bi Y, Yang H, Chen X, Liu H, Lu Y, et al. mTOR limits the recruitment of $\mathrm{CD} 11 \mathrm{~b}+\mathrm{Gr} 1+\mathrm{Ly} 6 \mathrm{Chigh}$ myeloid-derived suppressor cells in protecting against murine immunological hepatic injury. J Leukoc Biol. (2014) 95:961-70. doi: $10.1189 / \mathrm{jlb} .0913473$

23. Lin Y, Wang B, Shan W, Tan Y, Feng J, Xu L, et al. mTOR inhibitor rapamycin induce polymorphonuclear myeloid-derived suppressor cells mobilization and function in protecting against acute graft-versus-host disease after bone marrow transplantation. Clin Immunol. (2018) 187:122-31. doi: 10.1016/j.clim.2017.11.005 
24. Wu T, Zhao Y, Wang H, Li Y, Shao L, Wang R, et al. mTOR masters monocytic myeloid-derived suppressor cells in mice with allografts or tumors. Sci Rep. (2016) 6:20250. doi: 10.1038/srep20250

25. Ochando JC, Krieger NR, Bromberg JS. Direct versus indirect allorecognition: visualization of dendritic cell distribution and interactions during rejection and tolerization. Am J Transplant. (2006) 6:2488-96. doi: 10.1111/j.1600-6143.2006.01494.x

26. Zhuang Q, Liu Q, Divito SJ, Zeng Q, Yatim KM, Hughes AD, et al. Graftinfiltrating host dendritic cells play a key role in organ transplant rejection. Nat Commun. (2016) 7:12623. doi: 10.1038/ncomms12623

27. Liu Q, Rojas-Canales DM, Divito SJ, Shufesky WJ, Stolz DB, Erdos G, et al. Donor dendritic cell-derived exosomes promote allograft-targeting immune response. J Clin Invest. (2016) 126:2805-20. doi: 10.1172/JCI84577

28. Zecher D, van Rooijen N, Rothstein DM, Shlomchik WD, Lakkis FG. An innate response to allogeneic nonself mediated by monocytes. I Immunol. (2009) 183:7810-6. doi: 10.4049/jimmunol.0902194

29. Oberbarnscheidt MH, Zeng Q, Li Q, Dai H, Williams AL, Shlomchik WD, et al. Non-self recognition by monocytes initiates allograft rejection. J Clin Invest. (2014) 124:3579-89. doi: 10.1172/JCI74370

30. Wyburn KR, Jose MD, Wu H, Atkins RC, Chadban SJ. The role of macrophages in allograft rejection. Transplantation. (2005) 80:1641-7. doi: 10.1097/01.tp.0000173903.26886.20

31. Hancock WW, Thomson NM, Atkins RC. Composition of interstitial cellular infiltrate identified by monoclonal antibodies in renal biopsies of rejecting human renal allografts. Transplantation. (1983) 35:458-63. doi: 10.1097/00007890-198305000-00013

32. Braza MS, van Leent MMT, Lameijer M, Sanchez-Gaytan BL, Arts RJW, Perez-Medina C, et al. Inhibiting inflammation with myeloid cell-specific nanobiologics promotes organ transplant acceptance. Immunity. (2018) 49:819-828 e6. doi: 10.1016/j.immuni.2018.09.008

33. Scozzi D, Ibrahim M, Menna C, Krupnick AS, Kreisel D, Gelman AE. The role of neutrophils in transplanted organs. Am J Transplant. (2017) 17:328-335. doi: 10.1111/ajt.13940

34. Scapini P, Lapinet-Vera JA, Gasperini S, Calzetti F, Bazzoni F, Cassatella MA. The neutrophil as a cellular source of chemokines. Immunol Rev. (2000) 177:195-203. doi: 10.1034/j.1600-065X.2000.17706.x

35. Puga I, Cols M, Barra CM, He B, Cassis L, Gentile M, et al. B cell-helper neutrophils stimulate the diversification and production of immunoglobulin in the marginal zone of the spleen. Nat Immunol. (2011) 13:170-80. doi: 10.1038/ni.2194

36. Vono M, Lin A, Norrby-Teglund A, Koup RA, Liang F, Lore K. Neutrophils acquire the capacity for antigen presentation to memory CD4(+) T cells in vitro and ex vivo. Blood. (2017) 129:1991-2001. doi: 10.1182/blood-2016-10-744441

37. Dugast AS, Haudebourg T, Coulon F, Heslan M, Haspot F, Poirier N, et al. Myeloid-derived suppressor cells accumulate in kidney allograft tolerance and specifically suppress effector T cell expansion. J Immunol. (2008) 180:7898906. doi: 10.4049/jimmunol.180.12.7898

38. Mazzoni A, Bronte V, Visintin A, Spitzer JH, Apolloni E, Serafini P, et al. Myeloid suppressor lines inhibit $\mathrm{T}$ cell responses by an NO-dependent mechanism. J Immunol. (2002) 168:689-95. doi: 10.4049/jimmunol.168.2.689

39. Dilek N, Vuillefroy de Silly R, Blancho G, Vanhove B. Myeloidderived suppressor cells: mechanisms of action and recent advances in their role in transplant tolerance. Front Immunol. (2012) 3:208. doi: 10.3389/fimmu.2012.00208

40. Dilek N, Poirier N, Usal C, Martinet B, Blancho G, Vanhove B. Control of transplant tolerance and intragraft regulatory $\mathrm{T}$ cell localization by myeloid-derived suppressor cells and CCL5. J Immunol. (2012) 188:4209-16. doi: 10.4049/jimmunol.1101512

41. Hock BD, Mackenzie KA, Cross NB, Taylor KG, Currie MJ, Robinson BA, et al. Renal transplant recipients have elevated frequencies of circulating myeloid-derived suppressor cells. Nephrol Dialy Transplant. (2012) 27:40210. doi: $10.1093 / \mathrm{ndt} / \mathrm{gfr} 264$

42. Hock BD, McKenzie JL, Cross NB, Currie MJ. Dynamic changes in myeloid derived suppressor cell subsets following renal transplant: a prospective study. Transplant Immunol. (2015) 32:164-71. doi: 10.1016/j.trim.2015.05.001

43. Luan Y, Mosheir E, Menon MC, Wilson D, Woytovich C, Ochando J, et al. Monocytic myeloid-derived suppressor cells accumulate in renal transplant patients and mediate CD4(+) Foxp3(+) Treg expansion. Am J Transplant. (2013) 13:3123-31. doi: 10.1111/ajt.12461

44. Hoechst B, Ormandy LA, Ballmaier M, Lehner F, Kruger C, Manns MP, et al. A new population of myeloid-derived suppressor cells in hepatocellular carcinoma patients induces CD4(+)CD25(+)Foxp3(+) T cells. Gastroenterology. (2008) 135:234-43. doi: 10.1053/j.gastro.2008.03.020

45. Hoechst B, Gamrekelashvili J, Manns MP, Greten TF, Korangy F. Plasticity of human Th17 cells and iTregs is orchestrated by different subsets of myeloid cells. Blood. (2011) 117:6532-41. doi: 10.1182/blood-2010-11-317321

46. Rekers NV, Bajema IM, Mallat MJ, Petersen B, Anholts JD, Swings GM, et al. Beneficial immune effects of myeloid-related proteins in kidney transplant rejection. Am J Transplant. (2016) 16:1441-55. doi: 10.1111/ajt.13634

47. Meng F, Chen S, Guo X, Chen Z, Huang X, Lai Y, et al. Clinical significance of myeloid-derived suppressor cells in human renal transplantation with acute T cell-mediated rejection. Inflammation. (2014) 37:1799-805. doi: 10.1007/s10753-014-9910-5

48. He Y, Bei J, Zeng H, Pan Z. The roles of sepsis-induced myeloid derived suppressor cells in mice corneal, skin and combined transplantation. Transplant Immunol. (2016) 34:8-13. doi: 10.1016/j.trim.2015.12.003

49. Lee HJ, Park SY, Jeong HJ, Kim HJ, Kim MK, Oh JY. Glucocorticoids induce corneal allograft tolerance through expansion of monocytic myeloidderived suppressor cells. Am J Transplant. (2018) 18:3029-37. doi: 10.1111/ajt. 15026

50. Wei C, Wang Y, Ma L, Wang X, Chi H, Zhang S, et al. Rapamycin nanomicelle ophthalmic solution reduces corneal allograft rejection by potentiating myeloid-derived suppressor cells' function. Front Immunol. (2018) 9:2283. doi: 10.3389/fimmu.2018.02283

51. Marigo I, Bosio E, Solito S, Mesa C, Fernandez A, Dolcetti L, et al. Tumor-induced tolerance and immune suppression depend on the C/EBPbeta transcription factor. Immunity. (2010) 32:790-802. doi: 10.1016/j.immuni.2010.05.010

52. Qin J, Arakawa Y, Morita M, Fung JJ, Qian S, Lu L. C-C Chemokine receptor type 2-dependent migration of myeloid-derived suppressor cells in protection of islet transplants. Transplantation. (2017) 101:1793-800. doi: 10.1097/TP.0000000000001529

53. Chou HS, Hsieh CC, Yang HR, Wang L, Arakawa Y, Brown K, et al. Hepatic stellate cells regulate immune response by way of induction of myeloid suppressor cells in mice. Hepatology. (2011) 53:1007-19. doi: $10.1002 /$ hep. 24162

54. Chou HS, Hsieh CC, Charles R, Wang L, Wagner T, Fung JJ, et al. Myeloid-derived suppressor cells protect islet transplants by B7-H1 mediated enhancement of T regulatory cells. Transplantation. (2012) 93:272-82. doi: 10.1097/TP.0b013e31823ffd39

55. Hsieh CC, Chou HS, Yang HR, Lin F, Bhatt S, Qin J, et al. The role of complement component 3 (C3) in differentiation of myeloid-derived suppressor cells. Blood. (2013) 121:1760-8. doi: 10.1182/blood-2012-06-440214

56. Gao X, Liu H, He B, Fu Z. Resistance to streptozotocin-induced autoimmune diabetes in absence of complement C3: myeloid-derived suppressor cells play a role. PloS ONE. (2013) 8:e66334. doi: 10.1371/journal.pone.0066334

57. Zhang W, Liang $\mathrm{S}$, Wu J, Horuzsko A. Human inhibitory receptor immunoglobulin-like transcript 2 amplifies CD11b+Gr1+ myeloidderived suppressor cells that promote long-term survival of allografts. Transplantation. (2008) 86:1125-34. doi: 10.1097/TP.0b013e318186fccd

58. De Wilde V, Van Rompaey N, Hill M, Lebrun JF, Lemaitre P, Lhomme F, et al. Endotoxin-induced myeloid-derived suppressor cells inhibit alloimmune responses via heme oxygenase-1. Am J Transplant. (2009) 9:2034-47. doi: 10.1111/j.1600-6143.2009.02757.x

59. Yamashita K, Ollinger R, McDaid J, Sakahama H, Wang H, Tyagi S, et al. Heme oxygenase-1 is essential for and promotes tolerance to transplanted organs. FASEB J. (2006) 20:776-8. doi: 10.1096/fj.05-4791fje

60. Yang F, Li Y, Wu T, Na N, Zhao Y, Li W, et al. TNFalpha-induced M-MDSCs promote transplant immune tolerance via nitric oxide. J Mol Med (Berl). (2016) 94:911-20. doi: 10.1007/s00109-016-1398-Z

61. Wu T, Sun C, Chen Z, Zhen Y, Peng J, Qi Z, et al. Smad3deficient CD11b(+)Gr1(+) myeloid-derived suppressor cells prevent allograft rejection via the nitric oxide pathway. J Immunol. (2012) 189:4989-5000. doi: 10.4049/jimmunol.1200068 
62. Liao J, Wang X, Bi Y, Shen B, Shao K, Yang H, et al. Dexamethasone potentiates myeloid-derived suppressor cell function in prolonging allograft survival through nitric oxide. J Leukoc Biol. (2014) 96:675-84. doi: 10.1189/jlb.2HI1113-611RR

63. Ochando JC, Conde P. Editorial: dexamethasone and MDSC in transplantation: yes to NO. J Leukoc Biol. (2014) 96:669-71. doi: 10.1189/jlb.3CE0514-272R

64. Gajardo T, Morales RA, Campos-Mora M, Campos-Acuna J, PinoLagos K. Exogenous interleukin-33 targets myeloid-derived suppressor cells and generates periphery-induced Foxp3 $(+)$ regulatory $\mathrm{T}$ cells in skin-transplanted mice. Immunology. (2015) 146:81-8. doi: 10.1111/imm. 12483

65. Garcia MR, Ledgerwood L, Yang Y, Xu J, Lal G, Burrell B, et al. Monocytic suppressive cells mediate cardiovascular transplantation tolerance in mice. $J$ Clin Invest. (2010) 120:2486-96. doi: 10.1172/JCI41628

66. Gong W, Ge F, Liu D, Wu Y, Liu F, Kim BS, et al. Role of myeloidderived suppressor cells in mouse pre-sensitized cardiac transplant model. Clin Immunol. (2014) 153:8-16. doi: 10.1016/j.clim.2014.03.013

67. Nakamura T, Nakao T, Yoshimura N, Ashihara E. Rapamycin prolongs cardiac allograft survival in a mouse model by inducing myeloid-derived suppressor cells. Am J Transplant. (2015) 15:2364-77. doi: 10.1111/ajt.13276

68. Nakamura T, Nakao T, Ashihara E, Yoshimura N. Myeloid-derived suppressor cells recruit $\mathrm{CD} 4(+) / \mathrm{Foxp} 3(+)$ regulatory $\mathrm{T}$ Cells in a murine cardiac allograft. Transplant Proc. (2016) 48:1275-8. doi: 10.1016/j.transproceed.2015.10.060

69. Kishimoto K, Yuan X, Auchincloss H, Jr. Sharpe AH, Mandelbrot DA, Sayegh MH. Mechanism of action of donor-specific transfusion in inducing tolerance: role of donor MHC molecules, donor co-stimulatory molecules, and indirect antigen presentation. J Am Soc Nephrol. (2004) 15:2423-8. doi: 10.1097/01.ASN.0000137883.20961.2D

70. Quezada SA, Fuller B, Jarvinen LZ, Gonzalez M, Blazar BR, Rudensky AY, et al. Mechanisms of donor-specific transfusion tolerance: preemptive induction of clonal T-cell exhaustion via indirect presentation. Blood. (2003) 102:1920-6. doi: 10.1182/blood-2003-02-0586

71. Bryant J, Lerret NM, Wang JJ, Kang HK, Tasch J, Zhang Z, et al. Preemptive donor apoptotic cell infusions induce IFN-gamma-producing myeloidderived suppressor cells for cardiac allograft protection. J Immunol. (2014) 192:6092-101. doi: 10.4049/jimmunol.1302771

72. Chen G, Kheradmand T, Bryant J, Wang S, Tasch J, Wang J, et al. Intragraft $\mathrm{CD} 11 \mathrm{~b}(+) \mathrm{IDO}(+)$ cells mediate cardiac allograft tolerance by ECDI-fixed donor splenocyte infusions. Am J Transplant. (2012) 12:2920-9. doi: 10.1111/j.1600-6143.2012.04203.x

73. Kamada N, Brons G, Davies HS. Fully allogeneic liver grafting in rats induces a state of systemic nonreactivity to donor transplantation antigens. Transplantation. (1980) 29:429-31. doi: 10.1097/00007890-19800500 0-00021

74. Qian S, Demetris AJ, Murase N, Rao AS, Fung JJ, Starzl TE. Murine liver allograft transplantation: tolerance and donor cell chimerism. Hepatology. (1994) 19:916-24. doi: 10.1002/hep.1840190418

75. Bumgardner GL, Li J, Heininger M, Orosz CG. Costimulation pathways in host immune responses to allogeneic hepatocytes. Transplantation. (1998) 66:1841-5. doi: 10.1097/00007890-199812270-00047
76. Yu MC, Chen CH, Liang X, Wang L, Gandhi CR, Fung JJ, et al. Inhibition of T-cell responses by hepatic stellate cells via B7-H1-mediated T-cell apoptosis in mice. Hepatology. (2004) 40:1312-21. doi: 10.1002/hep.20488

77. Kim KS, Lee JS, Choi GS, Kwon CHD, Cho JW, Lee SK, et al. Long-term outcomes after stent insertion in patients with early and late hepatic vein outflow obstruction after living donor liver transplantation. Ann Surg Treat Res. (2018) 95:333-9. doi: 10.4174/astr.2018.95.6.333

78. Hamdani S, Thiolat A, Naserian S, Grondin C, Moutereau S, Hulin A, et al. Delayed and short course of rapamycin prevents organ rejection after allogeneic liver transplantation in rats. World J Gastroenterol. (2017) 23:696272. doi: 10.3748/wjg.v23.i38.6962

79. Okano S, Abu-Elmagd K, Kish DD, Keslar K, Baldwin WM, 3rd, Fairchild RL, et al. Myeloid-derived suppressor cells increase and inhibit donor-reactive $\mathrm{T}$ cell responses to graft intestinal epithelium in intestinal transplant patients. Am J Transplant. (2018) 18:2544-58. doi: 10.1111/ajt.14718

80. Sharma NS, Wille KM, Athira S, Zhi D, Hough KP, Diaz-Guzman E, et al. Distal airway microbiome is associated with immunoregulatory myeloid cell responses in lung transplant recipients. J Heart Lung Transplant. (2017) 37:206-16. doi: 10.1016/j.healun.2017.07.007

81. Koehn BH, Apostolova P, Haverkamp JM, Miller JS, McCullar V, Tolar J, et al. GVHD-associated, inflammasome-mediated loss of function in adoptively transferred myeloid-derived suppressor cells. Blood. (2015) 126:1621-8. doi: 10.1182/blood-2015-03-634691

82. Cassetta L, Baekkevold ES, Brandau S, Bujko A, Cassatella MA, Dorhoi A, et al. Deciphering myeloid-derived suppressor cells: isolation and markers in humans, mice and non-human primates. Cancer Immunol Immunother. (2019). doi: 10.1007/s00262-019-02302-2. [Epub ahead of print].

83. Zhang G, Huang H, Zhu Y, Yu G, Gao X, Xu Y, et al. A novel subset of B7-H3(+)CD14(+)HLA-DR(-/low) myeloid-derived suppressor cells are associated with progression of human NSCLC. Oncoimmunology. (2015) 4:e977164. doi: 10.4161/2162402X.2014.977164

84. Fridlender ZG, Sun J, Mishalian I, Singhal S, Cheng G, Kapoor V, et al. Transcriptomic analysis comparing tumor-associated neutrophils with granulocytic myeloid-derived suppressor cells and normal neutrophils. PLoS ONE. (2012) 7:e31524. doi: 10.1371/journal.pone.0031524

85. Negorev D, Beier UH, Zhang T, Quatromoni JG, Bhojnagarwala P, Albelda $\mathrm{SM}$, et al. Human neutrophils can mimic myeloid-derived suppressor cells (PMN-MDSC) and suppress microbead or lectin-induced $\mathrm{T}$ cell proliferation through artefactual mechanisms. Sci Rep. (2018) 8:3135. doi: 10.1038/s41598-018-21450-6

Conflict of Interest Statement: The authors declare that the research was conducted in the absence of any commercial or financial relationships that could be construed as a potential conflict of interest.

Copyright (C) 2019 Ochando, Conde, Utrero-Rico and Paz-Artal. This is an openaccess article distributed under the terms of the Creative Commons Attribution License (CC BY). The use, distribution or reproduction in other forums is permitted, provided the original author(s) and the copyright owner(s) are credited and that the original publication in this journal is cited, in accordance with accepted academic practice. No use, distribution or reproduction is permitted which does not comply with these terms. 